Scientific Paper

\title{
Uncertainties in the measurement of relative doses in radiotherapy
}

\author{
Krzysztof BULIŃSKI ${ }^{\mathrm{a}, \mathrm{b}}$, Tomasz KUSZEWSKI ${ }^{\mathrm{a}, \mathrm{b}}$, Katarzyna WNUK ${ }^{\mathrm{a}, \mathrm{b}}$, Janusz BRAZIEWICZ ${ }^{\mathrm{a}, \mathrm{b}}$, Krzysztof ŚLOSAREK $^{\mathrm{c}, \mathrm{d},{ }^{*}}$ \\ ${ }^{a}$ Institute of Physics, Jan Kochanowski University, Kielce, Poland \\ ${ }^{b}$ Holycross Cancer Center, Kielce, Poland \\ ${ }^{c}$ Collegium Medicum, Jan Kochanowski University, Kielce, Poland \\ ${ }^{d}$ Maria Skłodowska - Curie, National Research Institute of Oncology, Gliwice Branch, Gliwice, Poland \\ *E-mail address: Krzysztof.Slosarek@io.gliwice.pl
}

\begin{abstract}
Both the measurement of the dose and the measurement of its distribution, like any other measurements, are subject to measurement uncertainties. These uncertainties affect all dose calculations and dose distributions in a patient's body during treatment planning in radiotherapy. Measurement uncertainty is not a medical physicist's error, but an inevitable element of their work. Planning the dose distribution in a patient's body, we often try to reduce it in the volume of critical organs (OaR - Organ at Risk) or increase the minimum dose in the PTV region by a few percent. It is believed that the measurement uncertainty should be taken into account in these calculations at the stage of treatment planning. The paper presents the method of calculating the measurement uncertainty for different physical quantities in radiotherapy as percentage depth dose, profile function and output factor, due to the fact that these quantities have a particular impact on the calculated dose distributions in a patient's body. The uncertainties that must be taken into account in planning treatment the planned dose per fraction and real in PTV, maybe different up to $4 \%$.
\end{abstract}

Key words: measurement uncertainty; standard dose; depth dose; beam profile; output factor.

\section{Introduction}

Clinical dosimetry is an extremely important part of the work of a medical physicist in radiotherapy. It can be divided into four stages. The first one is related to the acceptance measurements of therapeutic devices (linear accelerators). Measurement of the physical parameters required by the treatment planning system is the second step. The third one involves verification of the accelerator operating parameters performed after each service inspection. And the fourth stage is the daily (or weekly) measurements of linac working conditions. Each of these steps affects the calculated dose distribution although their influence on the final result (dose distribution) is different.

The acceptance tests performed after the installation of the therapeutic accelerator are undoubtedly the most important dosimetry measurements. It is because they are basic reference values for latter measurements.

The preparation of a set of measurements that are introduced into the treatment planning system in order to configure it and to model therapeutic beams is a crucial stage of clinical dosimetry. ${ }^{1}$ Imprecise measurements have an impact on all prepared treatment plans, which means that all treated patients may receive medical support inconsistent with the therapeutic goals.
Checking the accelerator operating parameters after the service inspection is in fact the adjustment of these parameters in such a way that all dosimetric conditions are exactly the same as on the day the linac was approved for clinical operation.

Daily (or weekly) tests are performed to check whether the accelerator works according to the values introduced into the planning system. Possible discrepancies will affect the difference between the measured dose and the calculated dose.

Each of these steps includes a measurement uncertainty that affects the calculated dose distribution. There may also happen a random error that can be eliminated by repeating measurements. However, it is a rare case that the measurements for example of percent depth doses are repeated several times because they are very time-consuming. Therefore, it is worth comparing the obtained values with other measurement data reported for other accelerators. If we do not have such data, we can use manufacturer settings or compare them with data measured in other centers. This will minimize the likelihood of a random error. However, it does not change the fact that the accuracy of the obtained measurement values is related to the amount of measurement uncertainty.

The paper presents the method of calculating the measurement uncertainty for different physical quantities in radiotherapy as depth dose, profile function and output factor, due to the fact that these quantities have a particular impact on the calculated dose distributions in a patient's body. 


\section{Measurement uncertainty}

It is well known that each measurement is subject to measurement uncertainty. The literature in this area is extensive (for example international recommendations and reports $^{2-4}$ ) but do not always reflect the intuitive meaning of these terms: error - uncertainty.

Each physical quantity has its real value " $\mathrm{X}$ ". This value is unknown to us and we have to measure it using appropriate instruments. Performing the measurement of this quantity we receive the value " $\mathrm{x}$ ". It is obvious that $\mathrm{X} \neq \mathrm{x}$ and we can make out the difference:

$\Delta X=X-x$

Eq. 1

The main problem is that we do not know the value of " $\mathrm{X}$ " (real quantity) and we also do not know how much the measured value "x" differs from the real quantity. For example, looking at different angles on the thermometer scale, one can read different values. We want to know the real value of " $\mathrm{X}$ " as accurately as possible because it is the main goal of our measurements. Performing only one measurement, we do not know how big an "error" we are making. To minimize the probability of making a big measurement error, typically we repeat the measurements several times. Taking " $n$ " independent measurements of the quantity " $\mathrm{X}$ ", we are able to receive the well-known arithmetic mean value:

$\bar{x}=\frac{1}{n} \sum_{i=1}^{n} x_{i}$

Eq. 2

$\bar{x}$ - arithmetic mean

$\mathrm{n}$ - number of measurements

$x_{i}$ - "i" measurement

Typically, we assume that such obtained value $\bar{x}$ (arithmetic mean of all measurements) is the real value of " $\mathrm{X}$ " or it is the closest to it. We use this value for further measurements or calculations. However, we have to remember that this quantity is subject to measurement uncertainty. As a possible consequence of repeated measurements, we eliminated coarse errors. The values obtained in each independent measurement will be slightly different. Let us consider an example of a temperature measurement that we have to include in the calculation of the dose or dose rate value measured in reference conditions. For example, we performed the measurement ten times (Table 1) with a thermometer which has a scale of $0.5^{\circ} \mathrm{C}$. We obtained a set of values that are "slightly" different. Outliers may be rejected if they deviate significantly from the other data.

To calculate the radiation dose, ${ }^{4-6}$ the knowledge of the atmospheric pressure is also required. To simplify the problem, we assume that we "know the exact" value of the pressure. We use both of these values to calculate the $k_{T, P}$ coefficient according to the following formula:
$k_{T P}=k_{T} \times k_{P}=\frac{273,15+T}{273,15+T_{0}} \times \frac{P_{0}}{P}$

where:

$k_{T}$ - temperature coefficient,

$k_{P}$ - pressure coefficient,

$T, T_{0}$ - the temperature at the time of the measurement and the reference value of temperature $\left(20^{\circ} \mathrm{C}\right)$,

$p, p_{0}-$ pressure at the time of the measurement and the reference value of pressure $1013 \mathrm{hPa}$ ).

The crucial question is: what is the influence of the temperature measurement uncertainty on the value of this coefficient?

The average of the measured temperature values from Table 1 is $21.9^{\circ} \mathrm{C}$. We want to know what is the smallest and largest measured temperature value. For this purpose, we calculate the measurement uncertainty.

And thus the "A" type measurement uncertainty ${ }^{7,8}$ is calculated using the formula for the standard deviation of the mean value $S_{\bar{T}}$ :

$\Delta T(A)=\sqrt{\frac{1}{n(n-1)} \sum_{i=1}^{n}\left(T_{i}-\bar{T}\right)^{2}}$

where:

$S_{\bar{T}}^{2}$ - temperature variance,

$\bar{T}$ - mean temperature,

$T_{i}$ - "i" temperature measurement,

$\mathrm{n}$ - number of measurements.

and it equals $0.1247^{\circ} \mathrm{C}$ for the measured values from Table 1.

The type "B" measurement uncertainty connected to the thermometer resolution uncertainty, which equals half of its scale $\left(\Delta \mathrm{t}=0.25^{\circ} \mathrm{C}\right)$, is calculated using the following formula:

$\Delta T(B)=\frac{\Delta t}{\sqrt{3}}=\sqrt{\frac{\Delta t^{2}}{3}}$

For "our" thermometer, the measurement uncertainty of "B" type equals $0.1443^{\circ} \mathrm{C}$. The uncertainty of the measured temperature is the root of the sum of squares of the absolute values of the uncertainties of "A" and "B" types as follows:

$\Delta T=\sqrt{\Delta T(A)^{2}+\Delta T(B)^{2}}$

Eq. 6

Putting the values into Equation 6, we get the measurement uncertainty equal to $\Delta \mathrm{T}=0.1908^{\circ} \mathrm{C}$.

Table 1. Examples of temperature values that have been used in the uncertainty calculations.

\begin{tabular}{cc}
\hline \hline Measurement & $\mathbf{T}_{\mathbf{i}}\left[{ }^{\circ} \mathbf{C}\right]$ \\
\hline 1 & 21.5 \\
2 & 22 \\
3 & 22.5 \\
4 & 21.5 \\
5 & 22 \\
6 & 21.5 \\
7 & 22.5 \\
8 & 21.5 \\
9 & 22 \\
10 & 22 \\
\hline \hline
\end{tabular}


We can add and subtract this value from the average temperature $\left(21.9^{\circ} \mathrm{C}\right)$ and we obtain the possible range of its variability, i.e. $22.09076^{\circ} \mathrm{C}-21.70924^{\circ} \mathrm{C}$. The true value of temperature can be found within this range. Inserting these cutoff points into Equation 3 we get the following possible range of the $\mathrm{k}_{\mathrm{T}}$ coefficient: $\mathrm{k}_{\mathrm{T}}=1.0071-1.0058$. In the same way, we should calculate the value range of the " $k_{\mathrm{P}}$ " coefficient. Because we take these factors into account in the formula for calculating the standard dose rate at reference condition. According to the law of propagation of uncertainty.

Similarly to the above, we can determine the limits of the variability of other parameters necessary to determine the radiation dose rate, i.e. the coefficients: calibration $\mathrm{N}_{\mathrm{D}, \mathrm{w}}$, recombination $\mathrm{k}_{\mathrm{s}}$, polarization $\mathrm{k}_{\mathrm{pol}}$, chamber position and beam quality $\mathrm{k}_{\mathrm{Q}, \mathrm{Q} 0}$. A detailed description is in the IAEA-TECDOC1585 report. Estimated on the basis of these factors uncertainty of the dose measurement is equal to approximately $1.5 \% .^{5}$

However, it should be remembered that by measuring such values as depth dose (DD), profile function (PF), wedge factor (WF) or output factor (OF), we measure the dose in two "places". For example, to determine DD we perform measurement at "g" depth and at the reference depth, for PF we measure doses at the " $x$ " distance from the beam axis and in the beam axis (Appendix 1). Thus, the measurement uncertainty of these quantities (DD, PF, OF) will be higher.

\section{Uncertainty in the dose per fraction determining}

Let's just suppose that we do not have a computerized treatment planning system (TPS), but we only have the measured values of the depth dose (DD), profile function (PF), output factor (OF) and we know the dose in reference conditions. In our example we are going to calculate the irradiation time (t) - Monitor Units (MU) for the dose per fraction $-2 \mathrm{~Gy}$ at a depth of $5 \mathrm{~cm}$ for reference parameters: beam size $(10 \times 10) \mathrm{cm}^{2}, \mathrm{SSD}=100 \mathrm{~cm}$ for the beam X6MV. For such estimation, we use the following formula: ${ }^{9}$

$$
t=\frac{d_{f}[G y]}{\dot{D}_{s t}\left[\frac{G y}{M U}\right] * D D * P F * O F}[M U]
$$

where:

$\mathrm{d}_{\mathrm{f}}-$ is a fractional dose $=2 \mathrm{~Gy}$

$\dot{D}_{\text {st }}$ - standard dose rate $=1 \mathrm{~Gy} / 100 \mathrm{MU}$

$D D$-depth dose: for depth of $5 \mathrm{~cm}$ equals 0.864 ,

$P F$ - profile function, in this case, we calculate the dose on the beam axis, therefore $=1$,

$O F$ - output factor for field size $(10 \times 10) \mathrm{cm}^{2}=1$.

Inserting these values into Equation 7 we get the final value for irradiation time $\mathrm{t}=231.5$ [MU].

Now, let's assume that a critical organ $(\mathrm{OaR})$ is located at the depth of $20 \mathrm{~cm}$ and $1 \mathrm{~cm}$ outside the field of the radiation beam. In this case we have: $\mathrm{DD}_{\mathrm{OaR}}=0.382 ; \mathrm{PF}_{\mathrm{OaR}}=0.14$ and $\mathrm{OF}=1$. So, we are able to calculate the dose that OaR will receive during one fraction of irradiation according to the following formula:

$d_{\text {fOaR }}[G y]=\dot{D}_{s t}\left[\frac{G y}{M U}\right] * D D * P F * O F * t[M U]$

Eq. 8

In our example, the dose of $\mathrm{OaR}$ equals $0.1238 \mathrm{~Gy}$ and is accepted by the doctor. For the sake of calculation simplification, in our estimations, we haven't taken into account the beam divergence and all the related consequences.

At this point, every medical physicist should ask: what is the uncertainty of the calculated value? Which is due to the uncertainty of dose measurement.

Figure 1 shows the dose drop in water along the beam axis as a function of depth dose (DD) and as a function of the distance from the beam axis (PF) together with marked measurement uncertainties.

We have to calculate the dose range in PTV and OaR regions. In this situation, we are talking only about point doses.

The "A" type measurement uncertainty for the fractional dose is calculated according to the well-known formula:

$\left|\Delta d_{f}\right|=\sum\left|\frac{\partial d_{f}}{\partial a}\right||\Delta a|$

Eq. 9

where: $\left|\frac{\partial d_{f}}{\partial a}\right|$ is the absolute value of the derivative of Equation 8 calculated for factors: $\dot{D}_{S T} ; D D ; F P ; O F$.
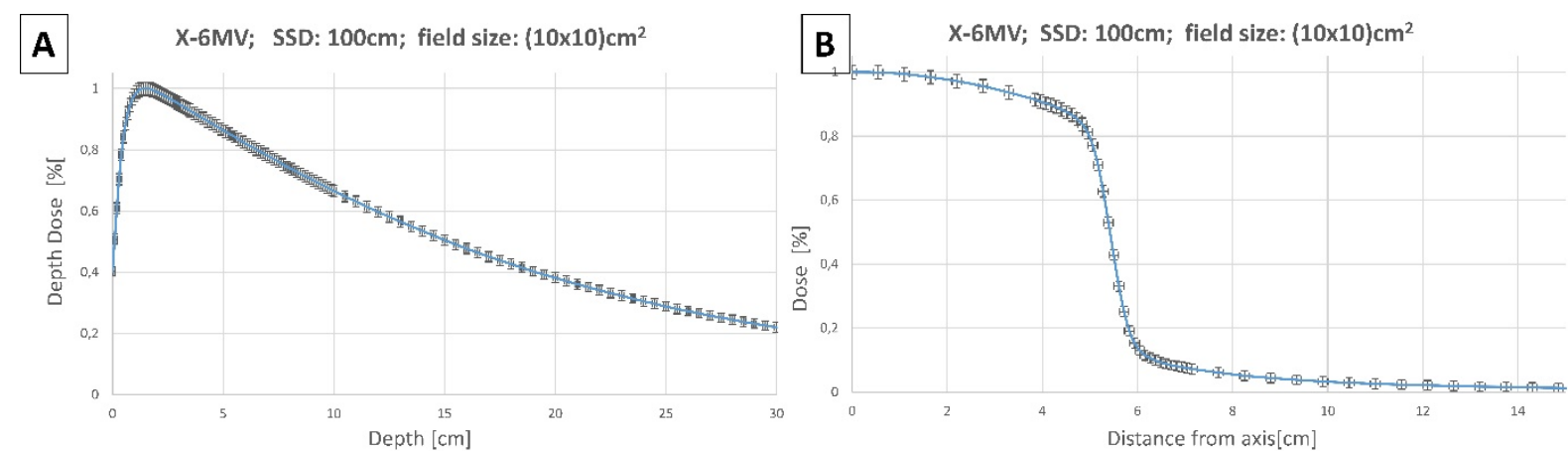

Figure 1. Dose decrease in the beam axis as a function of depth (A) and as a function of the distance from the beam axis (B) together with marked measurement uncertainties (Appendix 1) 
Finally, we receive:

$$
\Delta d_{f}=\sqrt{\begin{array}{c}
\left(\frac{d d_{f}}{d D_{S T}} \Delta D_{S T}\right)^{2}+\left(\frac{d d_{f}}{d D D} \Delta D D\right)^{2}+\left(\frac{d d_{f}}{d F P} \Delta P F\right)^{2} \\
+\left(\frac{d d_{f}}{d O F} \Delta O F\right)^{2}+\left(\frac{d d_{f}}{d M U} \Delta M U\right)^{2}
\end{array}}
$$

Taking into account estimated measurement uncertainty according to the above formula, the fractional dose in PTV region is within the range of $2 \mathrm{~Gy} \pm 0.0845 \mathrm{~Gy}$, which gives the relative uncertainty of $4.1 \%$ (we adopted $\Delta \mathrm{MU}$ as zero for simplification) (Appendix 2). This is a lot, but we have to remember that the measurement uncertainty of the standard dose (at reference conditions) has been defined as $1.5 \%$, and the fractional dose is calculated on the basis of the measured factors such as: depth dose, beam profile, output factors and others, which increase the overall error. However, if the dose per fraction that we plan to deliver in PTV region is calculated with $\sim 4 \%$ uncertainty, then we can and should ask a question: what will be the uncertainty of the dose administration in the critical organ? Similarly to the above, we can calculate the uncertainty of the dose administration in the critical organ and the final dose is in the range $0.1238 \mathrm{~Gy} \pm 0.0145 \mathrm{~Gy}$. Therefore, at this point, the relative uncertainty is equal to $11.7 \%$. In this case, the dose is very small, so the relative uncertainty has increased significantly. However, it may be relevant if the value of the maximum dose in OaR has been adopted as the maximum acceptable value. In such a situation, exceeding the total dose may be problematic. These uncertainties should be taken into account when we approve the treatment plan.

\section{Discussion}

The presented calculations of measurement uncertainty for the dose per fraction in PTV and OaR regions may be difficult to accept because in treatment plans we require much greater accuracy. This is the truth. However, we have the impression that medical physicists have deviated from the principles of measuring physical quantities. We are fascinated by computer algorithms, Monte Carlo computational methods, neural networks, artificial intelligence etc. but we forgot about the principles of measurements. We don't mean to say that we don't make them. Yes, we perform various types of measurements but the statistics of these measurements are of great influence on the final result. We set the measurement phantom as accurately as possible and take the measurement but usually once (they are time-consuming). Then we export the measured data in the required format (ASCII, txt, TPS manufacturer) to the treatment planning system. During the beam modeling procedure, depending on the version of the treatment planning system, we can demonstrate greater or lesser creativity to minimize the difference between the calculated and measured absolute doses and their distributions.

However, when making measurements of, for example, the profile function, when we see that some points "clearly" stand out from the rest, shouldn't the measurements be repeated?

Figure 2 shows example profile functions for different field dimensions. These are measured data that can be used to prepare calculation models.

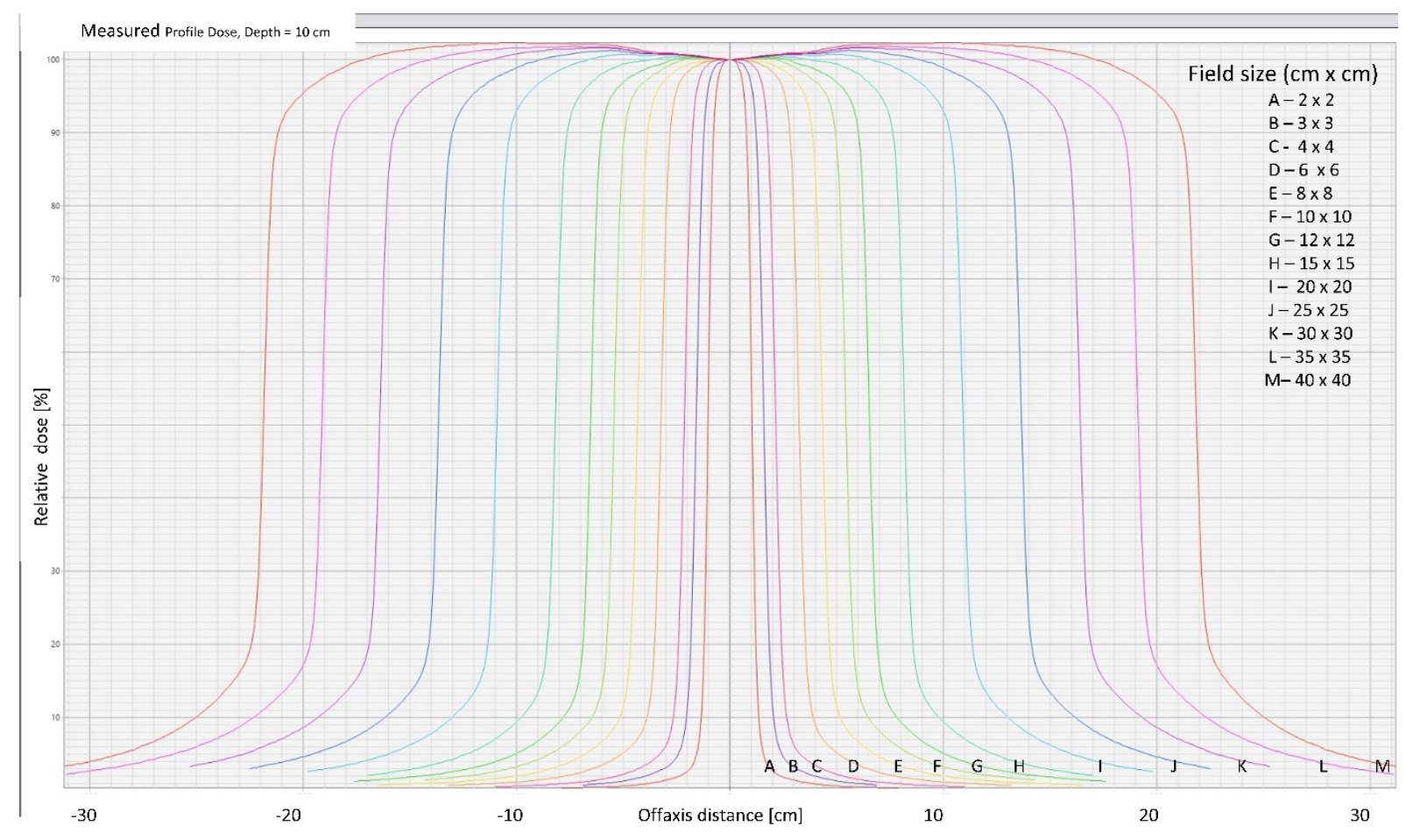

Figure 2. Examples of profile functions for different dimensions (from $2 \times 2 \mathrm{~cm}^{2}$ up to $40 \times 40 \mathrm{~cm}^{2}$ at the same depth (10 cm). They can be exported to the treatment planning system in the appropriate format. 


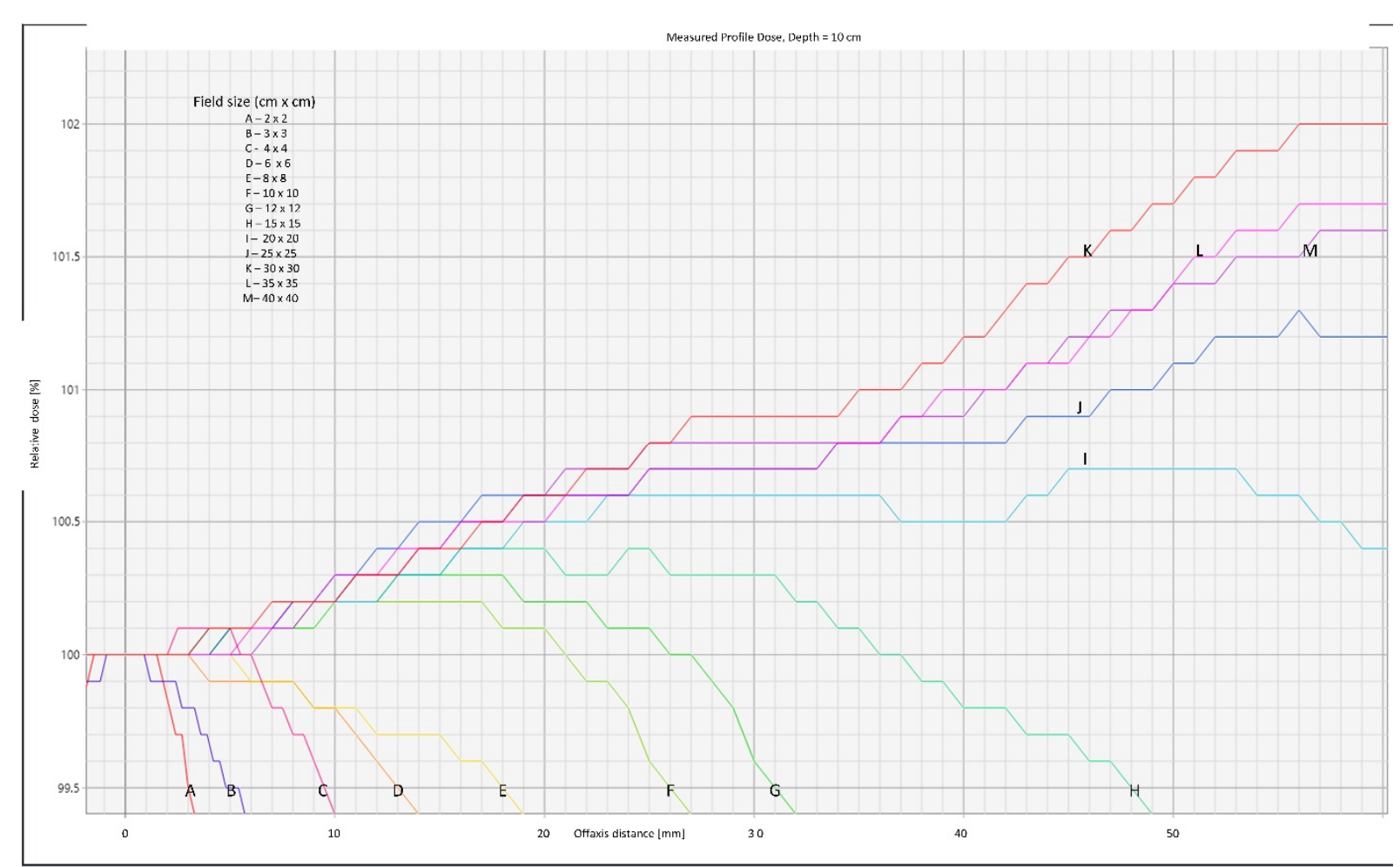

Figure 3. Enlarged ranges of the dose values of the profile function. It can be seen that the measurement values show differences in certain intervals, which may cause "disturbances" in the dose distribution calculated by the treatment planning system.

At first glance, it seems that the measurements have been made correctly. They are symmetric and there are no visible points that significantly "stand out" from the others. However, let us enlarge the range between $95-105 \%$ of the dose value and the distance from the beam axis up to $10 \mathrm{~cm}$ (see Figure 3).

Perhaps these results are within the measurement uncertainty that fluctuates around a few percent. It seems reasonable to assume that re-measuring could change this graph. It is true, that these are very small "fluctuations", but the dose calculated by us may (but does not have to) be burdened with the sum of "small" uncertainties.

The performed calculations of the measurement uncertainty show that they increase with the depth and distance from the beam axis and with the field size (Table 2 and Table 3). The $46 \%$ of uncertainty value (Table 2) for the dose at a distance of $10 \mathrm{~cm}$ from the beam axis, i.e. $5 \mathrm{~cm}$ beyond the beam edge, requires explanation. The dose at this point is very small. The relative measurement uncertainty increases with decreasing measurement value. If we assume that the average dose at this point is equal to $0.01 \mathrm{~Gy}$, then in fact it may be in the range of about $0.005-0.015 \mathrm{~Gy}$.

What could be the practical consequences of differences between the current dose deliver to a patient and the measured dose? Depending on the location and size of the treated tumor volumes, we use different dimensions of the radiation beams. In radiosurgery treatment we use small fields (measurement uncertainty of OF, Table 3, for a small field is greater than for a large field), critical organs are in close proximity to the PTV region (measurement uncertainty outside the field at a great depth, Table 2), and dose per fraction is large. Each shift of the patient relative to the position planned may enter a big difference between the calculated dose and dose delivery to a patient. Therefore, in radiosurgery, special attention should be paid to the precision of treatment because the summation of uncertainties may lead to clinically significant differences in the dose distribution.

Table 2. Measurement uncertainties calculated for DD and PF for different depths and distances from the beam axis. In a special case, at a depth of $30 \mathrm{~cm}$ and $10 \mathrm{~cm}$ from the beam axis, the uncertainty may exceed $40 \%$.

\begin{tabular}{cccc}
\hline \hline Depth [cm] & $\begin{array}{c}\text { DD uncertainty } \\
{[\%]}\end{array}$ & $\begin{array}{c}\text { Distance [cm] } \\
\text { from beam axis; } \\
\text { (field dimension 5cm) }\end{array}$ & $\begin{array}{c}\text { PF } \\
\text { Uncertainty } \\
{[\%]}\end{array}$ \\
\hline 0 & 4.01 & 0 & 2.12 \\
1.5 & 2.12 & 4 & 2.24 \\
5 & 2.29 & 5 & 2.42 \\
15 & 3.33 & 6 & 10.85 \\
30 & 6.98 & 10 & 46.62 \\
\hline \hline
\end{tabular}

Table 3. Uncertainty for the measurement of the OF coefficient for square beam field. It fluctuates around the value of $2 \%$.

\begin{tabular}{ccc}
\hline \hline Side of the beam [cm] & OF & Relative uncertainty [\%] \\
\hline 3 & 0.88 & 2.45 \\
4 & 0.91 & 2.36 \\
5 & 0.93 & 2.30 \\
7 & 0.96 & 2.21 \\
10 & 1 & 2.12 \\
15 & 1.04 & 2.04 \\
20 & 1.07 & 2.00 \\
30 & 1.10 & 1.94 \\
40 & 1.12 & 1.92 \\
\hline \hline
\end{tabular}




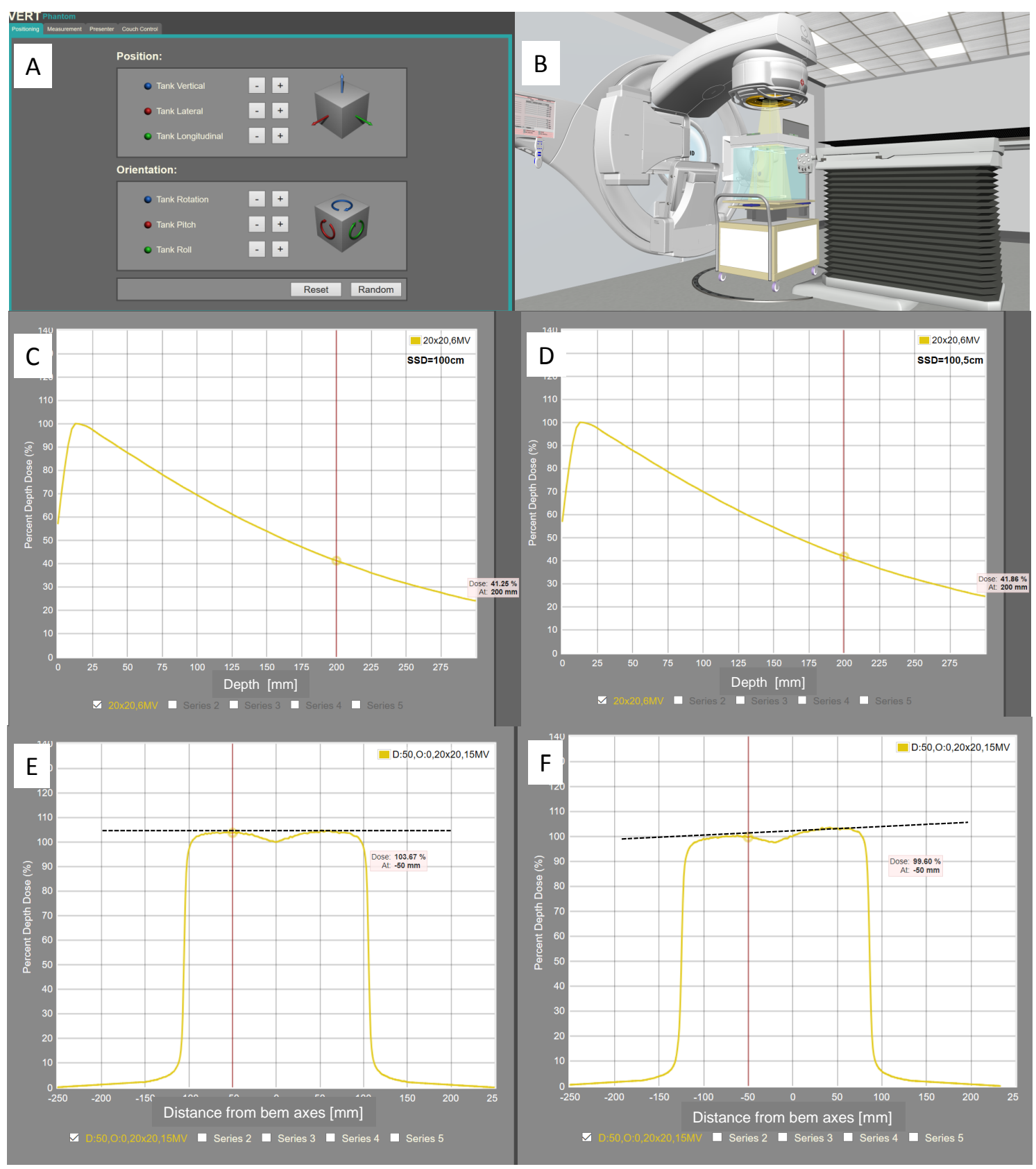

Figure 4. Virtual measurements performed with the use of VERT software (A) and module for setting the measurement phantom (B). Moving the phantom, changing the SSD from $100 \mathrm{~cm}$ (C) to $100.5 \mathrm{~cm}$ (D) changes the PDG, in this case, for X6MV beam and $20 \times 20 \mathrm{~cm}^{2}$ radiation area from $41.25 \%$ to $41.86 \%$. The imprecise positioning of the phantom causes the slope of the beam profile ( $E$ and $F$ ).

It is obvious that not every radiation dose will be given with a difference of several percent but, if the measurement data introduced into the treatment planning system include, due to the coincidence of unfavorable events, values with the maximum measurement uncertainty then each patient for whom the dose was calculated will receive a dose different from the planned one.

And yet the measurements we make are influenced not only by the measurement uncertainty associated with the (standard) dose measurement. We can imagine that the water surface of the measurement phantom is not perpendicular to the beam axis or the phantom distance is different from the defined one (Figure 4). Thus, the data introduced into the treatment planning system will have a significant impact on the results of the dose distribution calculations. It is worth adding that simulating this type of error during actual measurements requires access to a therapeutic device. The time of taking the measurements is also important. Meanwhile, by using the VERT software (installed at the Institute of Physics of Jan Kochanowski University in Kielce), we can simulate various situations that affect the results of dose measurements. An example of simulating the effect of a $0.5 \%$ SSD distance change and imprecise phantom setting on the DD size and dose profile is shown in Figure $\mathbf{4}$ which illustrates the seriousness of the measurement accuracy issue. Visualization of this type of issue is extremely important, especially for young medical physicists starting work in the field of radiotherapy. 


\section{Summary}

Dose measurements are subject to measurement uncertainties. There are two types of measurement uncertainties - "A" and "B" type that one can speak about. The former can be minimized through statistics, i.e. increasing the number of measurements. The "B" uncertainty can be reduced by increasing the accuracy of measurements, although the class of used measuring equipment has a large influence on this value. What is important, in our considerations we limited ourselves to determining only the doses and their distributions. We do not evaluate the effect of dose adjustment on the likelihood of local cure (TCP tumor control probability) or complications (NTCP normal tissue complication probability). For each neoplastic lesion and critical organs, a change in the physical dose influences the treatment outcomes in a different way. ${ }^{10}$ When discussing the dose distribution with a doctor, we should pay attention to the uncertainty of dose administration. ${ }^{11}$ It does not result from our error but it is a part of the measurement process and dose calculations. We also did not take into account the fundamental issue i.e. the precision of patient positioning which is a significant stage of the therapy. We can risk a statement, that may be the most important. If the patient is not placed on the therapeutic device table in accordance with the treatment plan, he will receive a different dose from the planned one. It does not change the fact that the measured dose amounts must be in accordance with the calculated uncertainty of the measurement.

\section{References}

1. Fraass B, Doppke K, Hunt M, et al. American Association of Physicists in Medicine Radiation Therapy Committee Task Group 53:Quality assurance for clinical radiotherapy treatment planning. Med Phys. 1998;25(10):1773-1829. https://doi.org/10.1118/1.598373

2. Szydłowski H. Niepewności w pomiarach. Międzynarodowe standardy w praktyce. Wydawnictwo Naukowe UAM, Poznań 2001.

3. International vocabulary of metrology - Basic and general concepts and associated terms (VIM). JCGM 200:2012. Available: https://www.bipm.org/en/publications/guides/vim.html

4. Almond PR, Biggs PJ, Coursey BM, et al. AAPM's TG-51 protocol for clinical reference dosimetry of high-energy photon and electron beams. Med Phys. 1999;26(9):1847-1870. https://doi.org/10.1118/1.598691

5. Absorbed Dose Determination in External Beam Radiotherapy: An International Code of Practice for Dosimetry based on Standards of Absorbed Dose to Water. IAEA TRS-398, 2000.

6. Code of Practice for the Absorbed Dose Determination in High Energy Photon and Electron Beams, Report 18 of the Netherlands Commission on Radiation Dosimetry. Revised edition. August 2012. https://doi.org/10.25030/ncs-018

7. Kubiaczyk A. Określenie niepewności pomiarów (poradnik do Laboratorium Fizyki). Wydział Fizyki, Politechnika Warszawska. Available: http://www.if.pw.edu.pl/\%7Elabfiz1p/cmsimple2_4/1instrukcje_pdf/ONP\%20-\%20poradnik.pdf

8. Evaluation of measurement data — Guide to the expression of uncertainty in measurement. JCGM 100:2008, 2008. Available: https://www.bipm.org/en/publications/guides/gum.html

9. $\quad$ Malicki J, Ślosarek K. Planowanie leczenia, dozymetria w radioterapii, Tom 2. Via Medica, 2018.

10. Moiseenko V, Battista J, Van Dyk J. Normal tissue complication probabilities: dependence on choice of biological model and dosevolume histogram reduction scheme. Int J Radiat Oncol Biol Phys. 2000;46:983-993. https://doi.org/10.1016/s0360-3016(99)00473-3

11. Hernandez V, Hansen CR, Widesott L, et al. What is quality in radiotherapy? The importance of evaluating dose metrics, complexity, and robustness of treatment plans. Radiother Oncol. 2020;153:26-33. https://doi.org/10.1016/j.radonc.2020.09.038 


\section{Appendix 1}

The "A" type measurement uncertainty for the " $\mathrm{X}$ " value is calculated according to the well-known formula:

1. $|\Delta X|=\sum\left|\frac{\partial X}{\partial a}\right||\Delta a|$

For $\left(\mathrm{DD}_{\mathrm{g}}\right)$ - at depth $(\mathrm{g})$ :

2. $\left|\Delta D D_{g}\right|=\left|\frac{1}{D}\right|\left|\Delta D_{g}\right|+\left|\frac{D_{g}}{D^{2}}\right||\Delta D|$

For $\left(P F_{x}\right)$ - at distance $(x)$ from axis :

3. $\left|\Delta P F_{x}\right|=\left|\frac{1}{D}\right|\left|\Delta D_{x}\right|+\left|\frac{D_{x}}{D^{2}}\right||\Delta D|$

For $\left(\mathrm{OF}_{\mathrm{s}}\right)$ - for beam size $(\mathrm{s})$ :

4. $\left|\Delta O F_{x s}\right|=\left|\frac{1}{D}\right|\left|\Delta D_{s}\right|+\left|\frac{D_{x}}{D^{2}}\right||\Delta D|$ 


\section{Appendix 2}

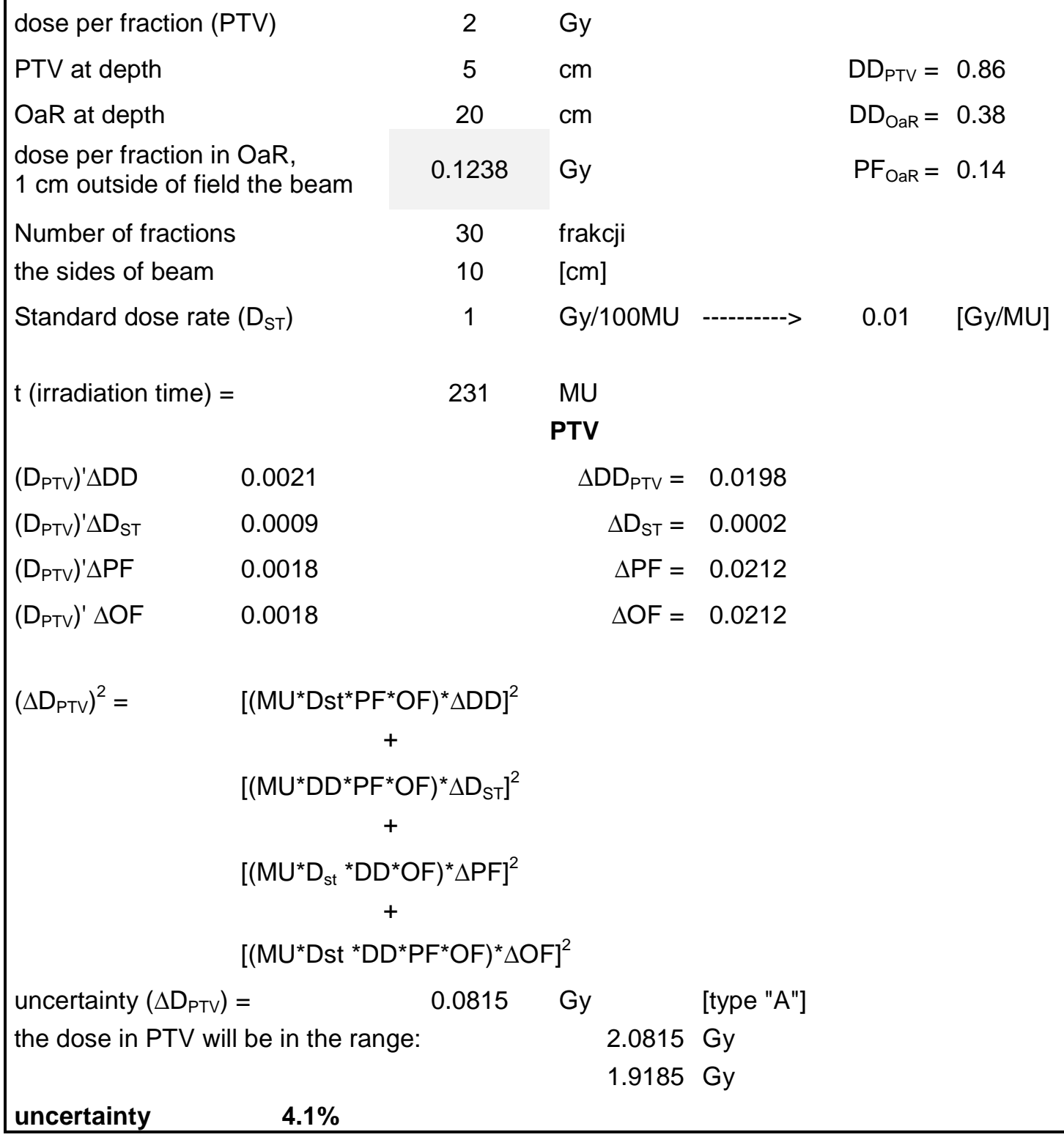

\begin{tabular}{llrl}
\hline & \multicolumn{2}{c}{ OaR } \\
$\left(\mathrm{D}_{\mathrm{OaR}}\right)^{\prime} \Delta \mathrm{DD}$ & 0.00002 & $\Delta \mathrm{DD}_{\mathrm{OaR}}=$ & 0.0161 \\
$\left(\mathrm{D}_{\mathrm{OaR}}\right)^{\prime} \Delta \mathrm{D}_{\mathrm{ST}}$ & 0.00000 & $\Delta \mathrm{D}_{\mathrm{ST}}=$ & 0.0002 \\
$\left(\mathrm{D}_{\mathrm{OaR}}\right)^{\prime} \Delta \mathrm{PF}$ & 0.00018 & $\Delta \mathrm{PF}=$ & 0.0151 \\
$\left(\mathrm{D}_{\mathrm{OaR}}\right)^{\prime} \Delta \mathrm{OF}$ & 0.00001 & $\Delta \mathrm{OF}=$ & 0.0212 \\
& & \\
uncertainty $\left(\Delta \mathrm{D}_{\mathrm{OaR}}\right)=$ & $0.0145 \mathrm{~Gy}$ \\
the dose in OaR will be in the range: & 0.1383 Gy \\
& $0.1093 \mathrm{~Gy}$ \\
uncertainty & $\mathbf{1 1 . 7 \%}$ & \\
\hline
\end{tabular}

